Supporting Information

\title{
Control of Photocarriers Separation and Recombination at Bismuth Oxyhalides Interface for Nitrogen Fixation
}

\author{
Yanyan Zhao, ${ }^{\dagger}$ Si Zhou, ${ }^{*}+, \star$ Jijun Zhao, ${ }^{\dagger}$ Yi Du,${ }^{\ddagger}$ and Shi Xue Dou ${ }^{\star}$ \\ ${ }^{\dagger}$ Key Laboratory of Materials Modification by Laser, Ion and Electron Beams (Dalian \\ University of Technology), Ministry of Education, Dalian 116024, China \\ †nstitute for Superconducting and Electronic Materials (ISEM), Australian Institute for \\ Innovative Materials (AIIM), University of Wollongong, Wollongong, NSW 2500, Australia
}

${ }^{*}$ Corresponding author. Tel: 86-0411-84706100, E-mail: sizhou@dlut.edu.cn (Si Zhou) 


\section{Computational methods}

Within the computational hydrogen electrode (CHE) model, the chemical potential of a proton-electron pair can be referred to the chemical potential of a gaseous $\mathrm{H}_{2}$ molecule. ${ }^{1}$ The Gibbs free energy of formation for each elementary step of $\mathrm{N}_{2}$ reduction is computed as

$$
\Delta G=\Delta E_{\mathrm{DFT}}+\Delta \mathrm{ZPE}-T \Delta S
$$

where $\Delta E_{\mathrm{DFT}}, \Delta \mathrm{ZPE}$ and $\Delta S$ are the differences in DFT total energy, zero-point energy and entropy of the two states before and after reaction, respectively; temperature $T$ is set to $300 \mathrm{~K}$ in our study. The $\Delta \mathrm{ZPE}$ and $\Delta S$ values of gaseous molecules were obtained from the NIST-JANAF thermodynamics table. ${ }^{2}$ For the reaction intermediates, we calculated their vibrational frequencies and derived $\Delta \mathrm{ZPE}$ and $\Delta S$ using the thermodynamics model within the harmonic approximation (Table S3 of Supporting Information).

The catalytic activity is characterized by the onset potential $U$, defined as the lowest potential (versus reversible hydrogen electrode, RHE) at which all the reaction steps are downhill in free energy, and equal to

$$
U=-\Delta G_{\max } / e
$$

where $\Delta G_{\max }$ is the maximum Gibbs free energy of formation among the reaction steps of $\mathrm{N}_{2}$ reduction; $e$ is the elementary charge.

The time-dependent $a b$ initio nonadiabatic molecular dynamics (NAMD) simulations implemented by the Hefei-NAMD code. ${ }^{3}$ After geometry optimization, we obtained the equilibrated configuration at $300 \mathrm{~K}$ by velocity rescaling method with Brillouin Zone (BZ) sampled by $\Gamma$ point only. The photocarrier relaxation process was simulated by the fewest switches surface hopping (FSSH) method. ${ }^{4}$ The decoherence induced surface hopping (DISH) method $^{5}$ was employed for the recombination process, where the energy difference between impurity states and band edges of BiOX is considerably large. After equilibration, the systems are heated to $300 \mathrm{~K}$ by velocity rescaling. Then, 2 ps and 1 ns microcanonical ab initio molecular dynamics trajectories with a time step of 1 fs were generated for the FSSH and DISH 
simulations, respectively. Using the molecular dynamics trajectory, the NAMD results were obtained by averaging over 50 different initial configurations. We sampled 5000 and 100 trajectories for last 2 ps (FSSH) and $1 \mathrm{~ns}$ (DISH), respectively. Considering the underestimation of band gap by the PBE functional, we corrected the Kohn-Sham states based on the energy levels using the Heyd-Scuseria-Ernzerhof (HSE06) hybrid functional implemented in NAMD simulation.

In the $a b$ initio NAMD simulation, ${ }^{3}$ the time-dependent charge density of the interacting system can be obtained from the time-dependent Kohn-Sham (TDKS) orbitals $\Psi_{p}(\mathbf{r}, t)$ as:

$$
\rho(\mathbf{r}, t)=\sum_{p=1}^{N_{e}}\left|\Psi_{p}(\mathbf{r}, t)\right|^{2}, p=1,2, \ldots, N_{\mathrm{e}}
$$

where $N_{\mathrm{e}}$ is the number of electrons. The time evolution of excited electron/hole carriers is described using TDKS equation

$$
\mathrm{i} \hbar \frac{\partial \Psi_{p}(\mathbf{r}, t)}{\partial t}=\mathcal{H}(\boldsymbol{r} ; \boldsymbol{R}(t)) \Psi_{p}(\boldsymbol{r}, t)
$$

Here $\Psi_{p}(\boldsymbol{r}, t)$ is the wave function of the excited electron/hole carriers and can be expanded by a set of KS orbitals

$$
\Psi_{p}(\mathbf{r}, t)=\sum_{k} c_{k}(t) \Phi_{k}(\mathbf{r} ; \mathbf{R}(t))
$$

Inserting Eq. S5 into Eq. S4 gives the equation of the expanding coefficients:

$$
\mathrm{i} \hbar \frac{\partial}{\partial t} c_{j}(t)=\sum_{k} c_{k}(t)\left(\varepsilon_{k}(t) \delta_{j k}+d_{j k}(t)\right)
$$

where $\varepsilon k$ is the energy of the adiabatic state $k$, and $d_{j k}$ is the non-adiabatic couplings (NAC) between the electronics states $j$ and $k$ :

$$
d_{\mathrm{jk}}=\left\langle\Phi_{j}\left|\frac{\partial}{\partial t}\right| \Phi_{k}\right\rangle=\sum_{I} \frac{\left\langle\Phi_{j}\left|\nabla_{\mathbf{R}_{I}} \mathcal{H}\right| \Phi_{k}\right\rangle}{\varepsilon_{k}-\varepsilon_{j}} \cdot \dot{\boldsymbol{R}}_{I}
$$

where $\mathcal{H}$ is the KS Hamiltonian; $\Phi_{j}, \Phi_{k}, \varepsilon_{j}$ and $\varepsilon_{k}$ are the wave functions and eigenvalues for electronic states $j$ and $k ; \dot{\boldsymbol{R}}_{I}$ is velocity of the nuclei. According to Eq. $\mathrm{S} 7$, the NAC mainly depends on the energy difference term $\left(\varepsilon k-\varepsilon_{j}\right)$, the electronphonon coupling term $\left\langle\Phi_{j}\left|\nabla_{\mathbf{R}_{I}} \mathcal{H}\right| \Phi_{k}\right\rangle$ and the nuclear velocity $\dot{\boldsymbol{R}}_{I}$.

The charge transfer of excited electron/hole carriers from region $\mathrm{A}$ to $\mathrm{B}$ is computed by integrating the electron/hole density over the region B as follows:

$$
\int \rho(\boldsymbol{r}, t) d \boldsymbol{r}=\int|\Psi(\mathbf{r}, t)|^{2} d \boldsymbol{r}=\sum_{i j} c_{k}{ }^{*}(t) c_{j}(t) \int \Phi_{i}{ }^{*}(\boldsymbol{r}, \boldsymbol{R}(t)) \Phi_{j}(\boldsymbol{r}, \boldsymbol{R}(t)) d \boldsymbol{r}
$$

Taking the time-derivative of Eq. S8 gives the expressions for adiabatic (AD) and non-adiabatic (NA) contributions to charge transfer: 


$$
\frac{d \int \rho(\boldsymbol{r}, t) d \boldsymbol{r}}{d t}=\sum_{i j}\left\{\frac{d\left(c_{i}{ }^{*} c_{j}\right)}{d t} \int \Phi_{i}{ }^{*} \Phi_{j} d \boldsymbol{r}+{c_{i}}^{*} c_{j} \frac{\int \Phi_{i}{ }^{*} \Phi_{j} d r}{d t}\right\}
$$

The first term on the right side is the nonadiabatic charge transfer due to change of state occupations of the adiabatic KS states; the second term is the adiabatic charge transfer due to the change of charge density of the KS adiabatic states. The sum of two terms gives the total charge transfer.

In our considered BiOX photocatalysts, electron (hole) transfer from the CBM (VBM) to the impurity states in Mo-BiOCl monolayer (process 1 and 2 in Figure 6a, b) has to overcome a considerable energy gap of $1.18(2.06) \mathrm{eV}$, so as for the electron-hole recombination in the $\mathrm{Mo}-\mathrm{BiOCl} / \mathrm{BiOBr}$ heterostructure (process 5 in Figure $6 \mathrm{a}, \mathrm{b})$. In such situations, the decoherence effect is prominent and we use the DISH algorithm to calculate the hopping probability of excited carriers. For the hole relaxation in the valence bands as well as the electron relaxation from conduction bands to the impurity states (that are very close to $\mathrm{CBM}$ ) in the $\mathrm{Mo}-\mathrm{BiOCl} / \mathrm{BiOBr}$ heterostructure (process 3 and 4 in Figure $6 \mathrm{a}, \mathrm{b}$ ), the FSSH method is adopted to simulate the intra-band transition of excited carriers.

For the DISH algorithm, the pure-dephasing time can be calculated by the linear response formalism. ${ }^{5}$ Given the time-dependent energies of the states $i$ and $j, E_{i}(t)$ and $E_{j}(t)$, the fluctuation of their difference is computed as ${ }^{6}$

$$
\delta E_{i j}(t)=\Delta E_{i j}(t)-\left\langle\Delta E_{i j}(t)\right\rangle
$$

where $\langle\cdot\rangle$ donates time-averaging and

$$
E_{i j}(t)=E_{i}(t)-E_{j}(t)
$$

The unnormalized autocorrelation function of the energy difference fluctuation

$$
C_{i j}(t)=\left\langle\delta E_{i j}\left(t^{\prime}\right) \delta E_{i j}\left(t-t^{\prime}\right)\right\rangle_{t^{\prime}}
$$

can then be used to compute the decoherence function

$$
D_{i j}(t)=\exp \left(-\frac{1}{\hbar^{2}} \int_{0}^{t} d t^{\prime} \int_{0}^{t^{\prime}} d t^{\prime \prime} C_{i j}\left(t^{\prime \prime}\right)\right)
$$

Fitting Eq. (S13) with a Gaussian function can obtain the pure dephasing time. The decoherence time $\left(\tau_{\alpha}\right)$ is related to the dephasing rate via

$$
\frac{1}{\tau_{\alpha}}(t)=\sum_{i \neq \alpha}^{N}\left|c_{i}(t)\right|^{2} r_{\alpha i}
$$

where $r_{\alpha \mathrm{i}}$ is the reciprocal of the dephasing time.

The decoherence time determined by DISH defines the time at which electronic coherences have dissolved due to interaction with the environment. A small 
decoherence time (or dephasing time) suppresses the capture of excited carriers and the subsequent electron-hole recombination. We computed the pure dephasing time for the electron and hole relaxation in $\mathrm{Mo}-\mathrm{BiOCl}$ monolayer (process 1 and 2 in Figure 6a, b), and electron-hole recombination in the $\mathrm{Mo}-\mathrm{BiOCl} / \mathrm{BiOBr}$ heterostructure (process 5 in Figure 6a, b), as presented in Table S6. 
Table S1. Formation energy $(\Delta H$, in unit of eV) and bond length $(d$, in unit of $\AA$ ) of $\mathrm{O}-\mathrm{M}$ of $3 d-5 d$ transitional metal (M) doped in $\mathrm{BiOCl}$ sheet, adsorption energy of a $\mathrm{N}_{2}$ molecule $\left(\Delta E_{\mathrm{N} 2} *\right.$, in unit of $\left.\mathrm{eV}\right)$ on the supercell of $3 \times 3$ and $4 \times 4 \mathrm{M}-\mathrm{BiOCl}$, adsorption energy of a $\mathrm{H}^{*}$ species $\left(\Delta E_{\mathrm{H}^{*}}\right.$, in unit of $\left.\mathrm{eV}\right)$ on the supercell of $3 \times 3 \mathrm{M}-\mathrm{BiOCl}$, bond length ( $d$, in unit of $\AA$ ) of $\mathrm{N}-\mathrm{N}$ and $\mathrm{N}-\mathrm{M}$. The activities of BiOCl upon doping do not change much with the doping level, as demonstrated by the binding energies of $\mathrm{N}_{2}$ molecules and $\mathrm{H}^{*}$ species for all the tested models.

\begin{tabular}{|c|c|c|c|c|c|c|c|c|}
\hline & $\mathrm{Sc}$ & $\mathrm{Ti}$ & $\mathrm{V}$ & $\mathrm{Cr}$ & $\mathrm{Mn}$ & $\mathrm{Fe}$ & Co & $\mathrm{Ni}$ \\
\hline$\Delta H$ & -9.09 & -7.51 & -6.07 & -4.33 & -4.06 & -3.48 & -2.97 & -2.42 \\
\hline$d(\mathrm{O}-\mathrm{M})$ & 2.15 & 2.03 & 2.08 & 1.94 & 1.91 & 1.99 & 1.88 & 1.86 \\
\hline $\begin{array}{l}\Delta \mathrm{E}_{\mathrm{N} 2} * \\
(3 \times 3)\end{array}$ & - & -0.11 & -0.34 & 0.25 & - & 0.04 & 0.12 & -0.03 \\
\hline $\begin{array}{l}\Delta \mathrm{E}_{\mathrm{N} 2 *} \\
(4 \times 4)\end{array}$ & - & -0.09 & -0.33 & -0.10 & - & -0.08 & -0.10 & -0.11 \\
\hline$\Delta \mathrm{E}_{\mathrm{H}^{*}}$ & - & -0.30 & 0.34 & 1.05 & - & 0.93 & 0.28 & -0.03 \\
\hline$d(\mathrm{~N}-\mathrm{N})$ & - & 1.13 & 1.12 & 1.12 & - & 1.11 & 1.11 & 1.11 \\
\hline \multirow[t]{2}{*}{$d(\mathrm{~N}-\mathrm{M})$} & - & 2.10 & 1.98 & 1.99 & - & 2.24 & 2.09 & 2.03 \\
\hline & $\mathrm{Cu}$ & $\mathrm{Y}$ & $\mathrm{Zr}$ & $\mathrm{Nb}$ & Mo & $\mathrm{Tc}$ & $\mathrm{Ru}$ & $\mathrm{Rh}$ \\
\hline$\Delta H$ & -0.84 & -10.03 & -9.95 & -7.40 & -4.63 & -3.96 & -3.99 & -2.13 \\
\hline$d(\mathrm{O}-\mathrm{M})$ & 1.87 & 2.33 & 2.15 & 2.14 & 2.20 & 2.13 & 2.25 & 2.01 \\
\hline $\begin{array}{l}\Delta \mathrm{E}_{\mathrm{N} 2} \\
(3 \times 3)\end{array}$ & - & - & - & -0.01 & -0.37 & - & -1.42 & -0.80 \\
\hline $\begin{array}{l}\Delta \mathrm{E}_{\mathrm{N} 2} * \\
(4 \times 4)\end{array}$ & - & - & - & -0.01 & -0.15 & - & -0.94 & -0.85 \\
\hline$\Delta \mathrm{E}_{\mathrm{H}^{*}}$ & - & - & - & -0.02 & 0.07 & - & -1.00 & -0.61 \\
\hline$d(\mathrm{~N}-\mathrm{N})$ & - & - & - & 1.15 & 1.14 & - & 1.13 & 1.12 \\
\hline \multirow[t]{2}{*}{$d(\mathrm{~N}-\mathrm{M})$} & - & - & - & 2.00 & 1.99 & - & 1.90 & 1.91 \\
\hline & $\mathrm{Pd}$ & $\mathrm{Hf}$ & $\mathrm{Ta}$ & W & $\operatorname{Re}$ & Os & $\mathrm{Ir}$ & $\mathrm{Pt}$ \\
\hline$\Delta H$ & -0.14 & -10.30 & -8.74 & -6.31 & -4.52 & -4.11 & -3.13 & -1.74 \\
\hline$d(\mathrm{O}-\mathrm{M})$ & 2.02 & 2.12 & 2.07 & 2.60 & 2.01 & 1.97 & 1.99 & 2.04 \\
\hline $\begin{array}{l}\Delta \mathrm{E}_{\mathrm{N} 2} \\
(3 \times 3)\end{array}$ & - & - & -0.16 & -0.34 & -0.55 & -1.16 & -1.15 & - \\
\hline $\begin{array}{l}\Delta \mathrm{E}_{\mathrm{N} 2} \\
(4 \times 4)\end{array}$ & - & - & -0.06 & -0.57 & -0.59 & -1.22 & -1.20 & - \\
\hline$\Delta \mathrm{E}_{\mathrm{H}^{*}}$ & - & - & -0.45 & -0.38 & -0.76 & -0.83 & -1.18 & - \\
\hline$d(\mathrm{~N}-\mathrm{N})$ & - & - & 1.16 & 1.16 & 1.15 & 1.14 & 1.13 & - \\
\hline$d(\mathrm{~N}-\mathrm{M})$ & - & - & 1.96 & 1.91 & 1.90 & 1.87 & 1.87 & - \\
\hline
\end{tabular}


Table S2. Adsorption energy (in unit of $\mathrm{eV}$ ) of a $\mathrm{N}_{2}$ molecule $\left(\Delta E_{\mathrm{N} 2}\right)$ and $\mathrm{H}^{*}$ species $\left(\Delta E_{\mathrm{H}^{*}}\right)$ on the $3 d-5 d$ transitional metal $(\mathrm{M})$ dopants in $\mathrm{BiOBr}$ sheet.

\begin{tabular}{ccccccccc}
\hline \hline & $\mathrm{Ti}$ & $\mathrm{V}$ & $\mathrm{Cr}$ & $\mathrm{Fe}$ & $\mathrm{Co}$ & $\mathrm{Ni}$ & $\mathrm{Nb}$ & $\mathrm{Mo}$ \\
$\Delta \mathrm{E}_{\mathrm{N} 2}$ & 0.46 & -0.03 & 0.10 & 0.64 & 0.34 & 0.30 & 0.24 & -0.06 \\
$\Delta \mathrm{EH}^{*}$ & 0.03 & 0.32 & 0.61 & 0.49 & -1.55 & -0.01 & -0.11 & -0.06 \\
\hline & $\mathrm{Ru}$ & $\mathrm{Rh}$ & $\mathrm{Ta}$ & $\mathrm{W}$ & $\mathrm{Re}$ & $\mathrm{Os}$ & $\mathrm{Ir}$ & \\
$\Delta \mathrm{E}_{\mathrm{N} 2}$ & -0.57 & -0.47 & 0.12 & -0.70 & -1.28 & -0.86 & -0.81 & \\
$\Delta \mathrm{E}^{*}$ & -0.45 & -0.56 & -0.60 & -0.84 & -1.61 & -0.80 & -1.11 & \\
\hline
\end{tabular}


Table S3. Gibbs free energy of formation $(\Delta G)$ for each elementary steps of $\mathrm{N}_{2}$ reduction on $\mathrm{Mo}(\mathrm{V}, \mathrm{Nb}, \mathrm{Ru}, \mathrm{W})$ doped $\mathrm{BiOCl}$ monolayers.

\begin{tabular}{|c|c|c|c|c|c|c|}
\hline \multirow{2}{*}{ step } & \multirow{2}{*}{ Reaction } & \multicolumn{5}{|c|}{$\Delta G(\mathrm{eV})$} \\
\hline & & Mo & $\mathrm{V}$ & $\mathrm{Nb}$ & $\mathrm{Ru}$ & $\mathrm{W}$ \\
\hline $\mathrm{R} 1$ & $\mathrm{~N}_{2} \rightarrow \mathrm{N}_{2} *$ & 0.38 & 0.21 & 0.52 & -0.40 & -0.04 \\
\hline $\mathrm{R} 2$ & $\mathrm{~N}_{2} *+\mathrm{H}^{+}+\mathrm{e}^{-} \rightarrow \mathrm{HN}_{2} *$ & 0.13 & 0.75 & 0.17 & 0.72 & -0.27 \\
\hline $\mathrm{R} 3$ & $\mathrm{HN}_{2} *+\mathrm{H}^{+}+\mathrm{e}^{-} \rightarrow \mathrm{H}_{2} \mathrm{~N}_{2} *$ & -0.73 & -0.76 & -0.95 & 0.29 & -0.64 \\
\hline $\mathrm{R} 4$ & $\mathrm{H}_{2} \mathrm{~N}_{2} *+\mathrm{H}^{+}+\mathrm{e}^{-} \rightarrow \mathrm{H}_{3} \mathrm{~N}_{2} *$ & 0.15 & 0.26 & 0.73 & -0.60 & -0.18 \\
\hline R5 & $\mathrm{H}_{3} \mathrm{~N}_{2} * \rightarrow \mathrm{N}^{*}$ & -0.64 & 1.12 & 0.58 & -0.45 & -0.64 \\
\hline R6 & $\mathrm{N}^{*}+\mathrm{H}^{+}+\mathrm{e}^{-} \rightarrow \mathrm{HN}^{*}$ & -0.53 & -2.29 & -2.52 & 0.54 & -0.40 \\
\hline R7 & $\mathrm{HN}^{*}+\mathrm{H}^{+}+\mathrm{e}^{-} \rightarrow \mathrm{H}_{2} \mathrm{~N}^{*}$ & 0.22 & -0.23 & 0.35 & -0.89 & 0.65 \\
\hline $\mathrm{R} 8$ & $\mathrm{H}_{2} \mathrm{~N}^{*}+\mathrm{H}^{+}+\mathrm{e}^{-} \rightarrow \mathrm{H}_{3} \mathrm{~N}^{*}$ & -0.23 & -0.47 & 0.47 & -0.25 & 0.26 \\
\hline R9 & $\mathrm{H}_{3} \mathrm{~N}^{*} \rightarrow \mathrm{NH}_{3}$ & 0.66 & 0.81 & 0.06 & 0.45 & 0.66 \\
\hline
\end{tabular}


Table S4. Gibbs free energy of formation $(\Delta G)$ for each elementary steps of $\mathrm{N}_{2}$ reduction on $\mathrm{Mo}(\mathrm{V}, \mathrm{Nb}, \mathrm{Ru}, \mathrm{W})$ doped $\mathrm{BiOBr}$ monolayers.

\begin{tabular}{|c|c|c|c|c|c|c|}
\hline \multirow{2}{*}{ step } & \multirow{2}{*}{ Reaction } & \multicolumn{5}{|c|}{$\Delta G(\mathrm{eV})$} \\
\hline & & Mo & $\mathrm{V}$ & $\mathrm{Nb}$ & $\mathrm{Ru}$ & $\mathrm{W}$ \\
\hline $\mathrm{R} 1$ & $\mathrm{~N}_{2} \rightarrow \mathrm{N}_{2} *$ & 0.47 & 0.51 & 0.77 & -0.04 & -0.17 \\
\hline $\mathrm{R} 2$ & $\mathrm{~N}_{2} *+\mathrm{H}^{+}+\mathrm{e}^{-} \rightarrow \mathrm{HN}_{2} *$ & 0.09 & 0.72 & 0.14 & 0.71 & -0.28 \\
\hline $\mathrm{R} 3$ & $\mathrm{HN}_{2} *+\mathrm{H}^{+}+\mathrm{e}^{-} \rightarrow \mathrm{H}_{2} \mathrm{~N}_{2} *$ & -0.71 & -0.74 & -0.93 & 0.29 & -0.64 \\
\hline $\mathrm{R} 4$ & $\mathrm{H}_{2} \mathrm{~N}_{2} *+\mathrm{H}^{+}+\mathrm{e}^{-} \rightarrow \mathrm{H}_{3} \mathrm{~N}_{2} *$ & -0.02 & 0.23 & 0.57 & -0.78 & -0.31 \\
\hline R5 & $\mathrm{H}_{3} \mathrm{~N}_{2} * \rightarrow \mathrm{N}^{*}$ & -0.64 & 0.70 & 0.44 & -0.46 & -0.64 \\
\hline R6 & $\mathrm{N}^{*}+\mathrm{H}^{+}+\mathrm{e}^{-} \rightarrow \mathrm{HN}^{*}$ & -0.45 & -1.99 & -2.30 & 0.58 & -0.32 \\
\hline R7 & $\mathrm{HN}^{*}+\mathrm{H}^{+}+\mathrm{e}^{-} \rightarrow \mathrm{H}_{2} \mathrm{~N}^{*}$ & 0.28 & -0.11 & 0.35 & -0.67 & 0.63 \\
\hline $\mathrm{R} 8$ & $\mathrm{H}_{2} \mathrm{~N}^{*}+\mathrm{H}^{+}+\mathrm{e}^{-} \rightarrow \mathrm{H}_{3} \mathrm{~N}^{*}$ & -0.20 & -0.59 & 0.49 & -1.26 & 0.26 \\
\hline R9 & $\mathrm{H}_{3} \mathrm{~N}^{*} \rightarrow \mathrm{NH}_{3}$ & 0.58 & 0.67 & -0.14 & 1.05 & 0.86 \\
\hline
\end{tabular}


Table S5. Zero-point energy (ZPE) and entropic correction (TS) at $T=300 \mathrm{~K}$ for the molecules and intermediate species involved in $\mathrm{N}_{2}$ reduction on transitional metal doped BiOX sheets. The ZPE and TS values of gaseous molecules were obtained from the NIST-JANAF thermodynamics table. ${ }^{2}$ For the reaction intermediates, we calculated their vibrational frequencies and derived ZPE and TS using the thermodynamics model within the harmonic approximation. ${ }^{7}$ The $(\mathrm{ZPE}-T S)$ values of intermediates are very similar for Mo-BiOCl monolayer.

\begin{tabular}{cccc}
\hline \hline Species & ZPE $(\mathrm{eV})$ & $T S(\mathrm{eV})$ & $\mathrm{ZPE}-T S(\mathrm{eV})$ \\
\hline $\mathrm{H}_{2}$ & 0.29 & 0.41 & -0.12 \\
$\mathrm{~N}_{2}$ & 0.16 & 0.60 & -0.44 \\
$\mathrm{~N}_{2}{ }^{*}$ & 0.21 & 0.12 & 0.09 \\
$\mathrm{HN}_{2}{ }^{*}$ & 0.50 & 0.11 & 0.39 \\
$\mathrm{H}_{2} \mathrm{~N}_{2}{ }^{*}$ & 0.83 & 0.16 & 0.67 \\
$\mathrm{H}_{3} \mathrm{~N}_{2}{ }^{*}$ & 1.18 & 0.15 & 1.03 \\
$\mathrm{~N}^{*}$ & 0.10 & 0.04 & 0.06 \\
$\mathrm{HN}^{*}$ & 0.37 & 0.07 & 0.30 \\
$\mathrm{H}_{2} \mathrm{~N}^{*}$ & 0.67 & 0.07 & 0.60 \\
$\mathrm{H}_{3} \mathrm{~N}^{*}$ & 1.07 & 0.24 & 0.83 \\
$\mathrm{NH}_{3}$ & 0.94 & 0.60 & 0.34 \\
\hline
\end{tabular}


Table S6. Time scale $(\tau)$ and pure dephasing time for the photocarriers to transfer from valence or conduction bands to the impurity states for the Mo-BiOCl monolayer and $\mathrm{Mo}-\mathrm{BiOCl} / \mathrm{BiOBr}$ heterostructure, respectively.

\begin{tabular}{ccccc}
\hline & \multicolumn{2}{c}{ Pure dephasing time (fs) } & \multicolumn{2}{c}{$\tau(\mathrm{ns})$} \\
& Hole & Electron & Hole & Electron \\
Mo-BiOCl & 3.89 & 3.56 & 2 & 0.450 \\
$\mathrm{Mo-BiOCl} / \mathrm{BiOBr}$ & 2.96 & -- & 3 & -- \\
\hline
\end{tabular}


(a)

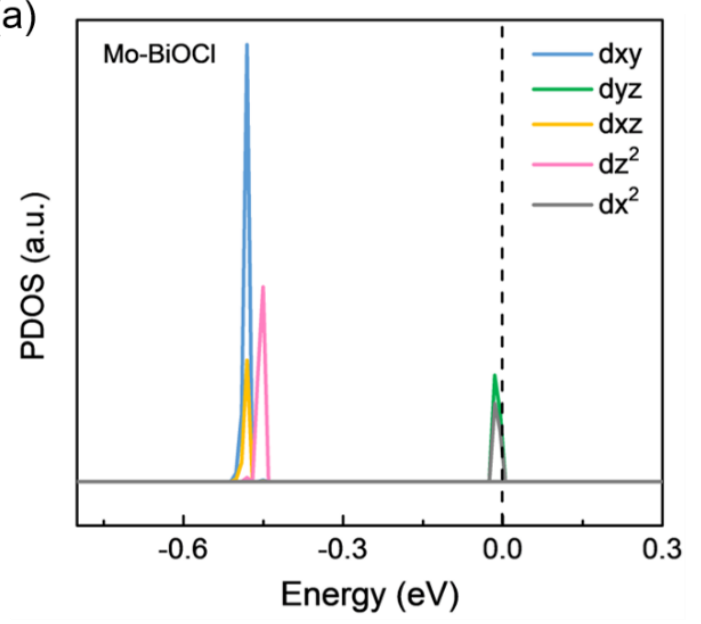

(b)

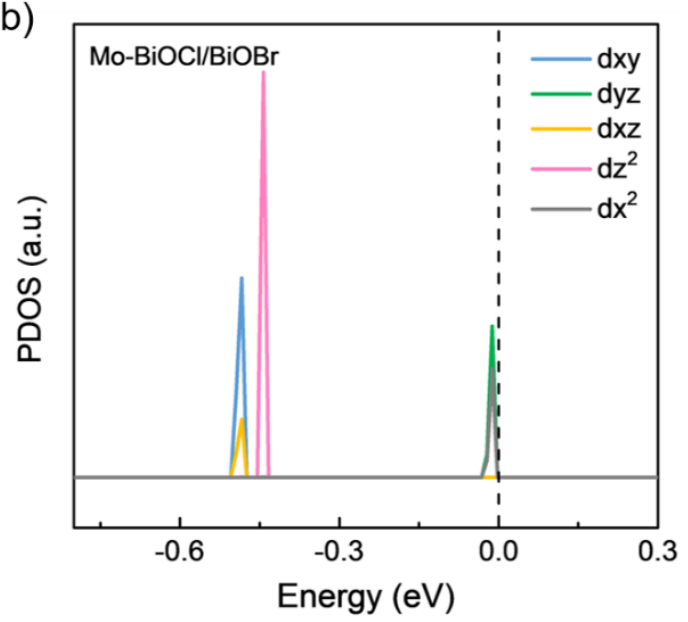

Figure S1. (a-b) Project density of states of d orbitals from Mo dopant in BiOCl monolayer and $\mathrm{BiOCl} / \mathrm{BiOBr}$ heterostructure, respectively. The dashed line shows the Fermi level that is shifted to zero. 
(a)

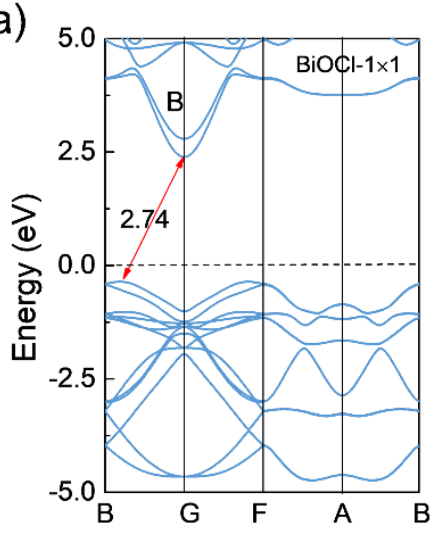

(b)

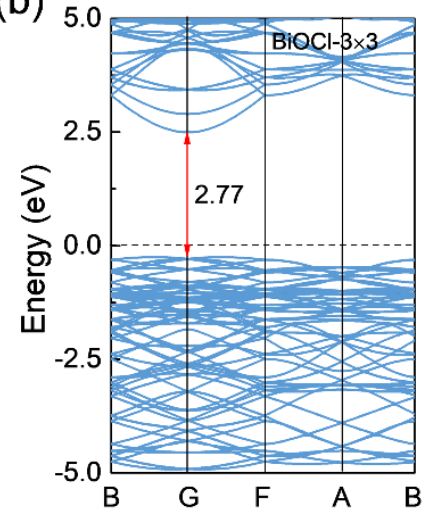

(c)

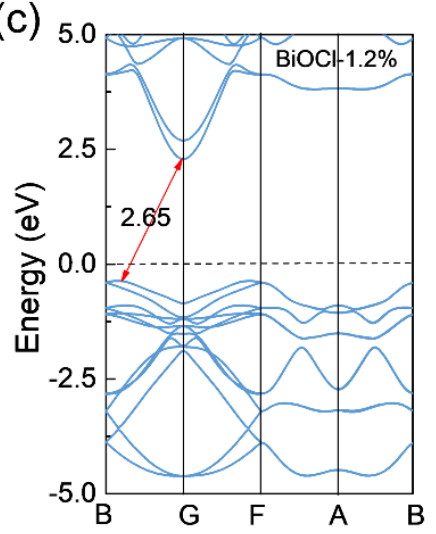

Figure S2. Electronic band structures of $\mathrm{BiOCl}$ monolayer using strain-free (a) unit cell $(1 \times 1)$ and (b) supercell (containing $3 \times 3$ unit cells), and (c) unit cell under 1.2\% extensive strain calculated by the PBE functional. 
(a)

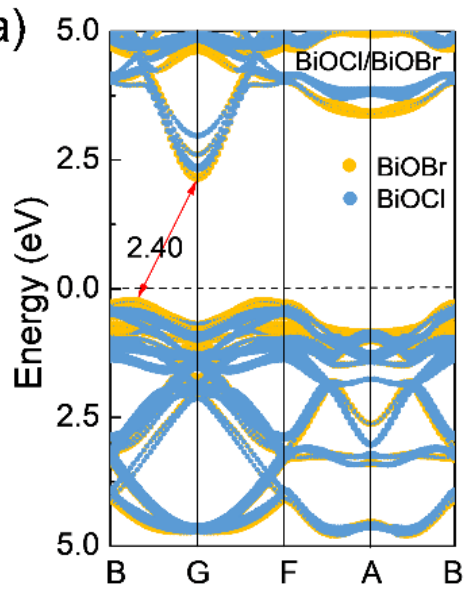

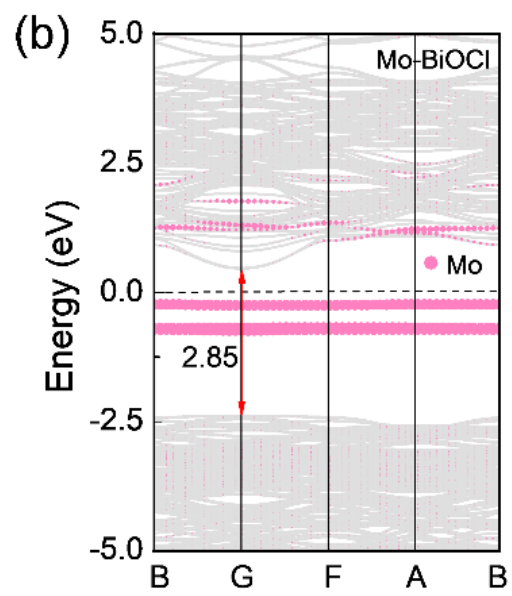

(c)

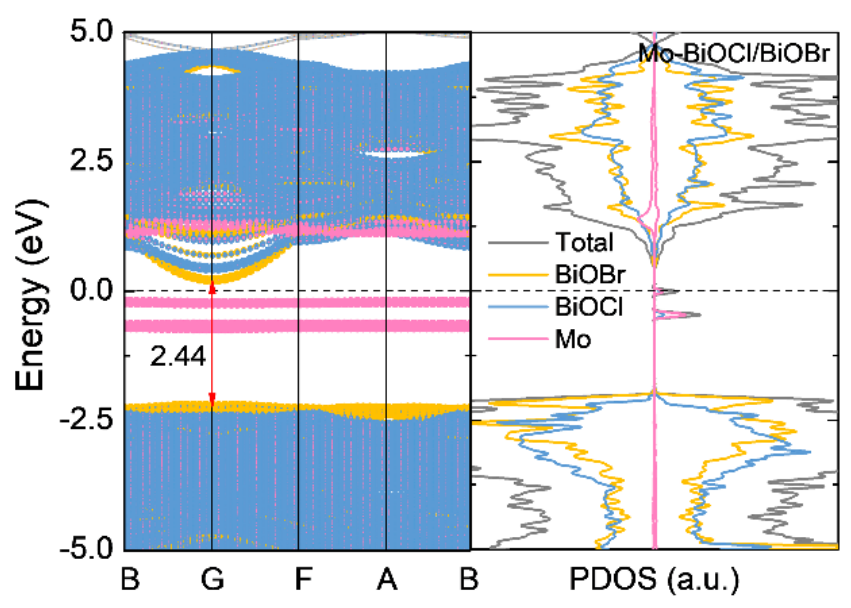

Figure S3. (a-b) Band structures of $\mathrm{BiOCl} / \mathrm{BiOBr}$ heterostructure and Mo-doped $\mathrm{BiOCl}$ monolayer, respectively, calculated using the PBE functional. (c) Band structure and local density of states (LDOS) of the Mo-BiOCl/BiOBr heterostructure. The dashed line shows the Fermi level that is shifted to zero. The black numbers indicate the band gap for each system. In (b) and (c), the impurity states from Mo atom are highlighted in pink. 
(a)

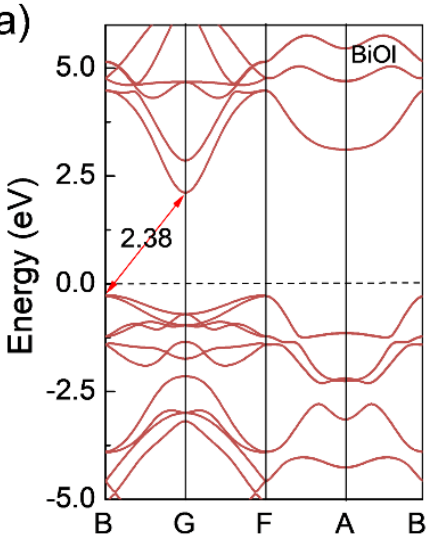

(b)

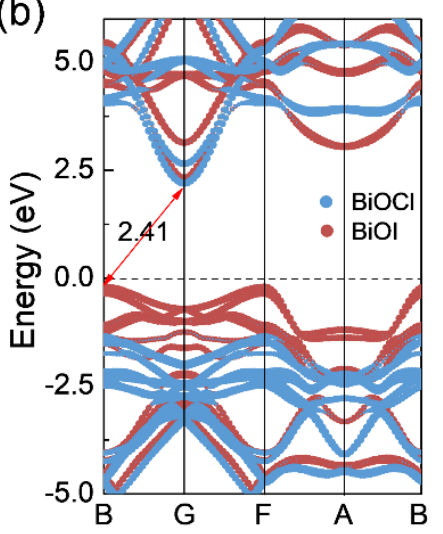

(c)

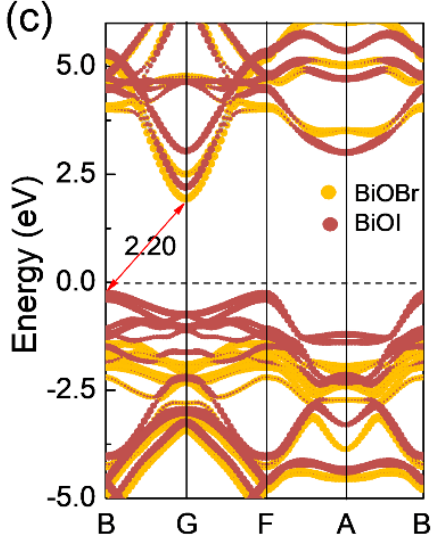

Figure S4. Band structures of (a) $\mathrm{BiOI}$ monolayer, (b) $\mathrm{BiOCl} / \mathrm{BiOI}$ and (c) $\mathrm{BiOBr} / \mathrm{BiOI}$ heterostructure, calculated using the HSE06 functional. The dashed line shows the Fermi level that is shifted to zero. The black numbers indicate the band gap for each system. 
(a)

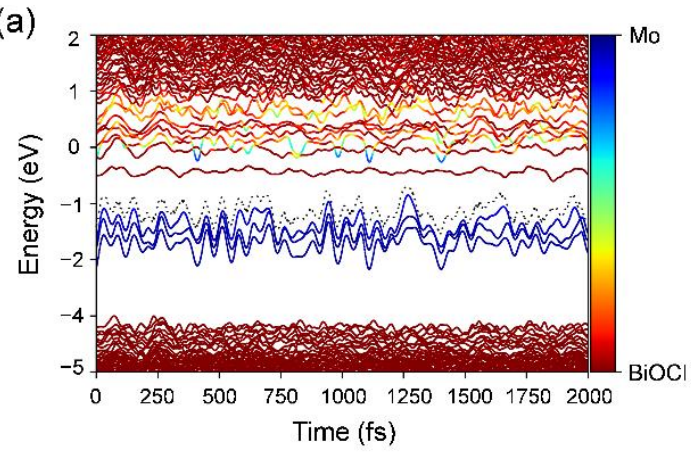

(b)

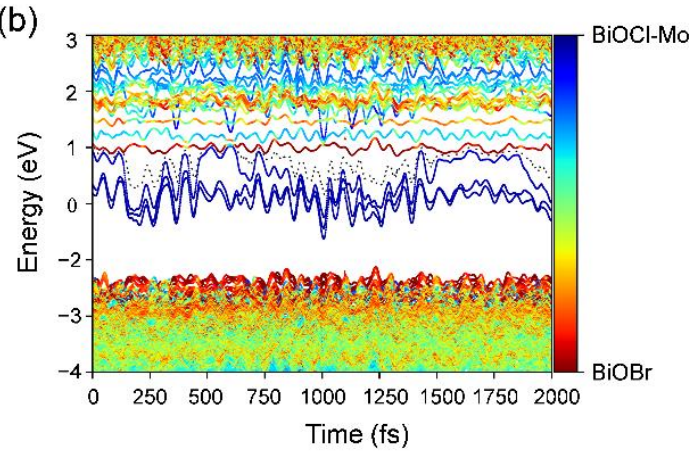

Figure S5. Time evolution of the energy states of (a) Mo-doped BiOCl monolayer and (b) $\mathrm{Mo}-\mathrm{BiOCl} / \mathrm{BiOBr}$ heterostructure in NAMD simulations using $\Gamma$-point calculation. The color indicates the spatial distribution of electronic states. The black dotted line shows time-dependent chemical potential. 
(a)

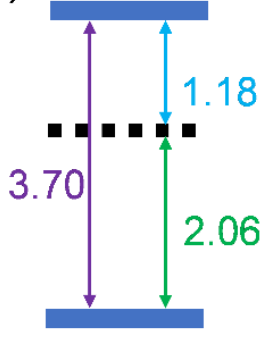

$\mathrm{Mo}-\mathrm{BiOCl}$ (b)

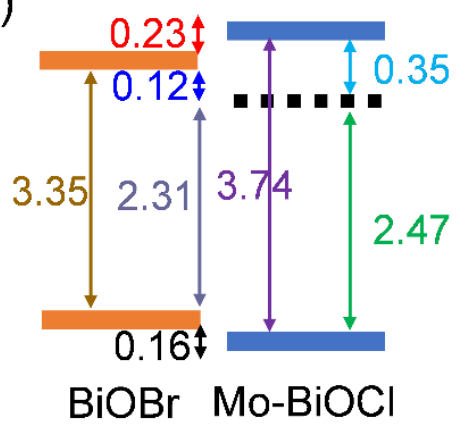

Figure S6. Schematic diagram of energy offset and gap involved in charge carrier relaxation and recombination for (a) $\mathrm{Mo}-\mathrm{BiOCl}$ monolayer and (b) $\mathrm{Mo}-\mathrm{BiOCl} / \mathrm{BiOBr}$ heterostructure at $\mathrm{T}=300 \mathrm{~K}(\mathrm{t}=0 \mathrm{fs})$, respectively. The black dashed line shows three impurity states from Mo dopant. The color numbers corresponding to the energy offset and gap marked by colored arrow, in unit of eV. 
(a)

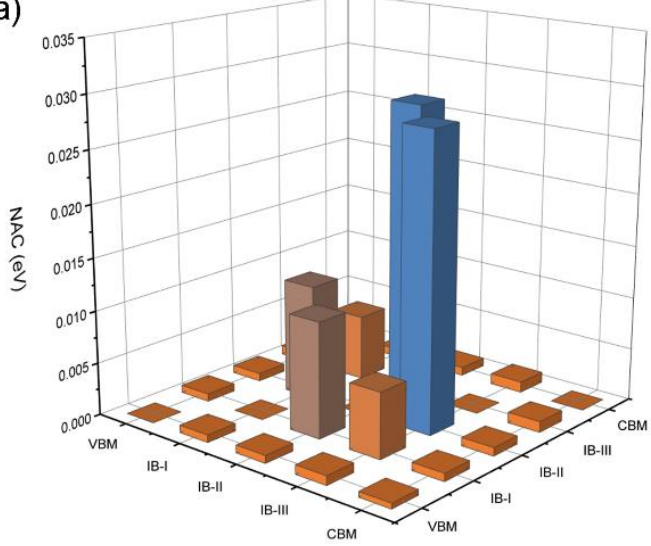

(b)

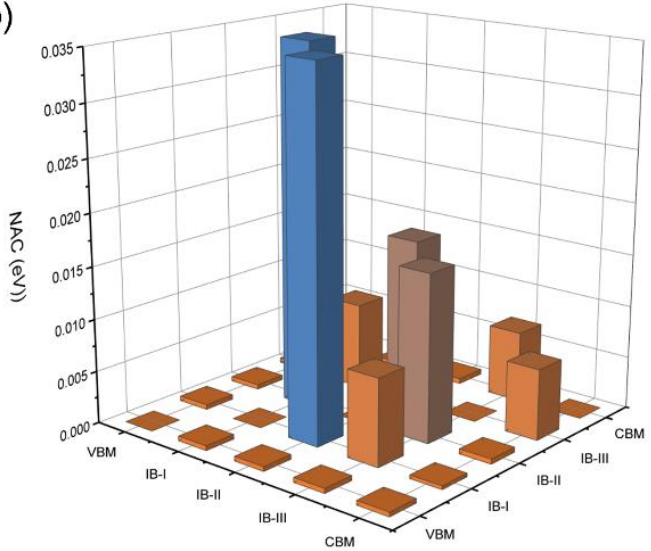

Figure S7. Averaged nonadiabatic coupling (NAC) elements between valence band maximum (or conduction band minimum) and impurity bands (IB) in (a) $\mathrm{Mo}-\mathrm{BiOCl}$ monolayer, and (b) $\mathrm{Mo}-\mathrm{BiOCl} / \mathrm{BiOBr}$ heterojunctions at $300 \mathrm{~K}$. 
(a)
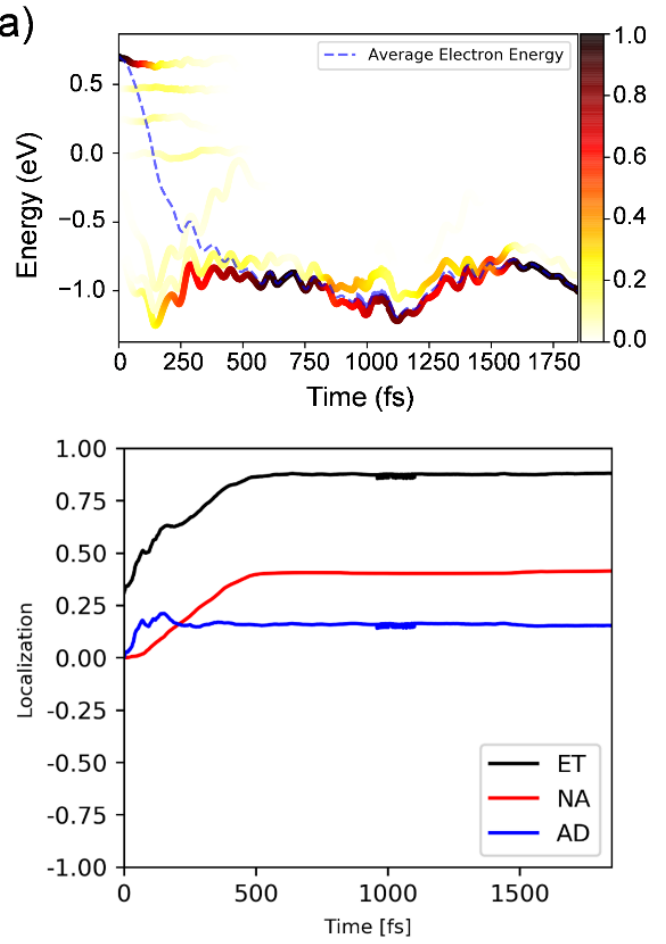

(b)
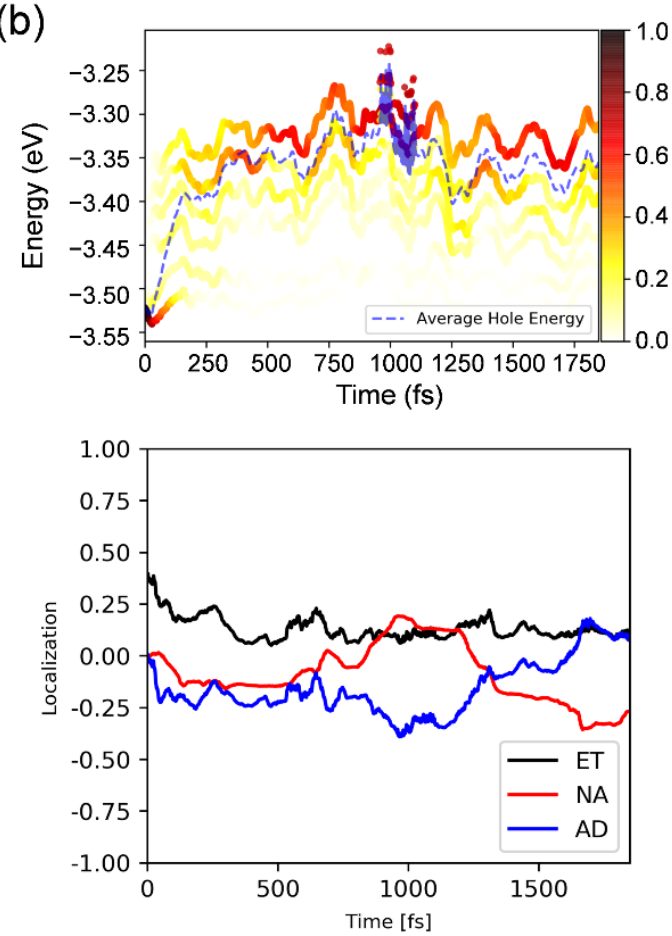

Figure S8. Top panels: time evolution of (a) electron and (b) hole relaxation in the $\mathrm{Mo}-\mathrm{BiOCl} / \mathrm{BiOBr}$ heterostructure. The color strips indicate the electron or hole distribution on different energy states, and the blue dashed line represents the averaged electron or hole energy. The energy reference is the Fermi level shifted to zero. The spatial localization of electron and hole carriers projected on the $\mathrm{Mo}-\mathrm{BiOCl}$ sheet are given by the bottom panels, showing the total electron transfer (ET), and the adiabatic (AD) and nonadiabatic (NA) contributions. The large fluctuation of hole energy and localization is due to the electron-phonon interaction at finite temperature, which can be further revealed by the Fourier transform of the phonon-induced fluctuations of VBM plotted in Figure S9. 


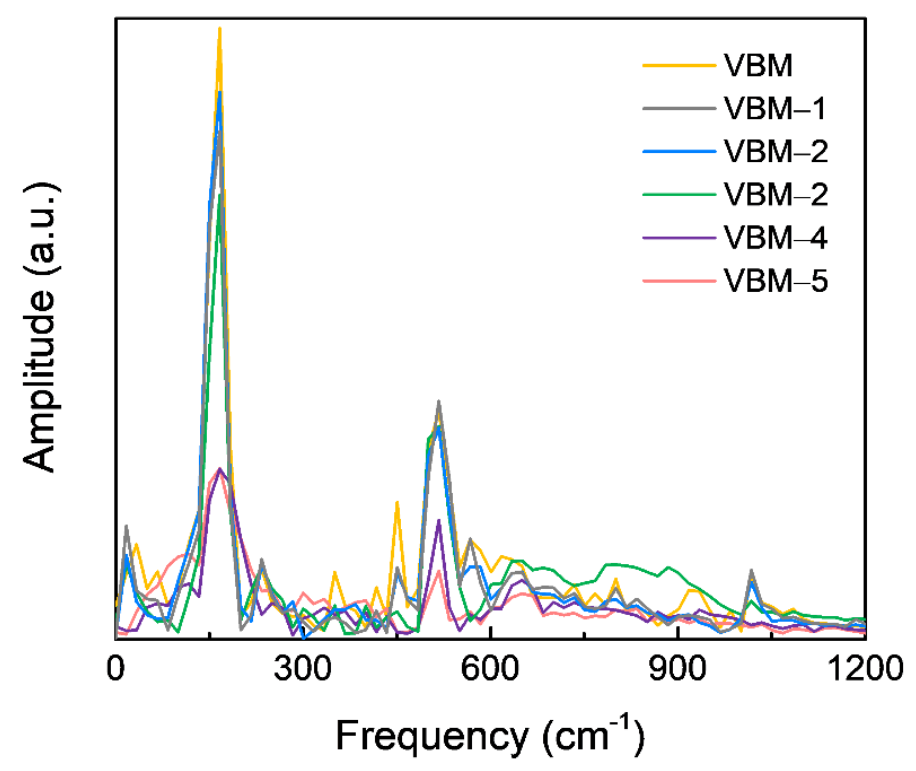

Figure S9. The Fourier transform spectra of the phonon-induced fluctuations from VBM to $\mathrm{VBM}-5$ for $\mathrm{Mo}-\mathrm{BiOCl} / \mathrm{BiOBr}$ heterostructure. 

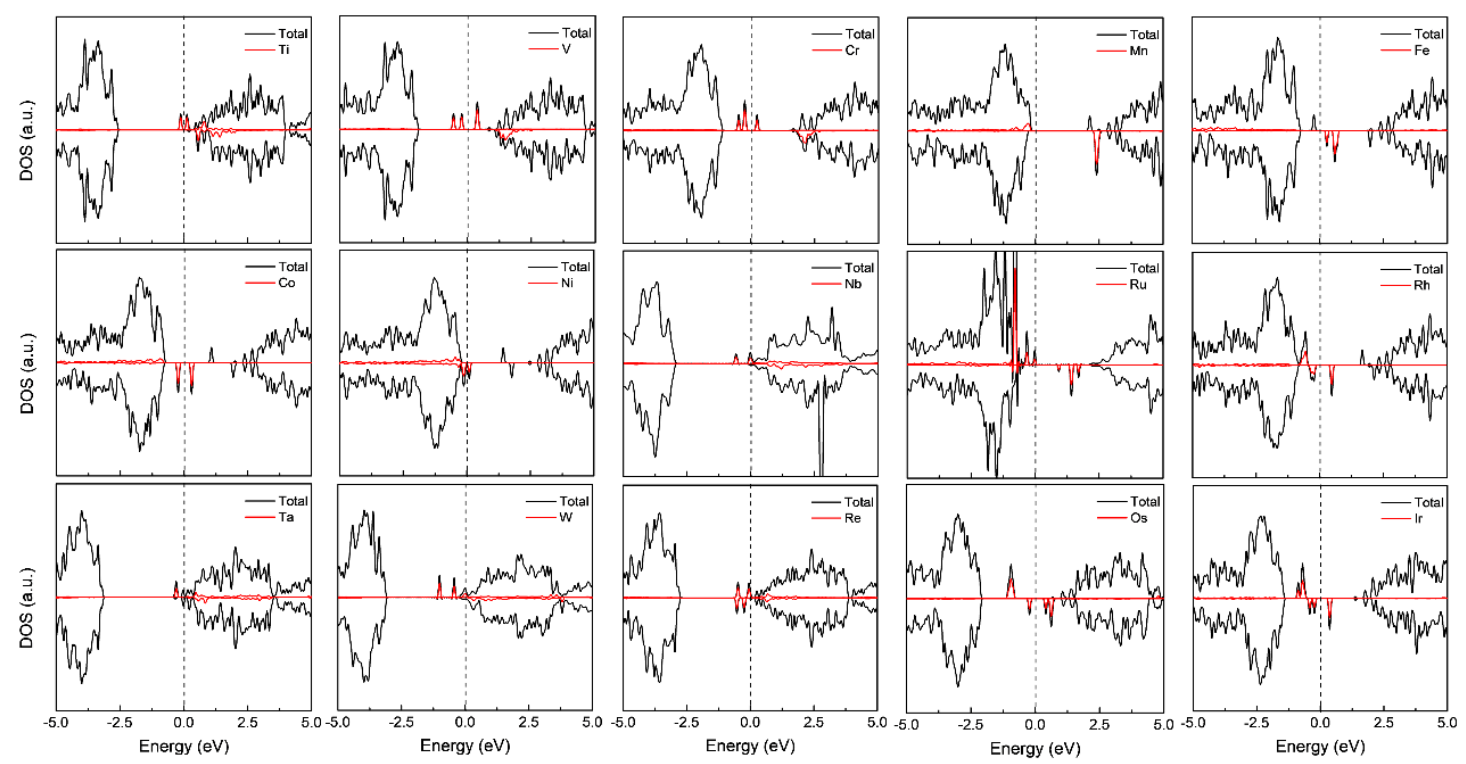

Figure S10. Density of states (DOS) of transitional metal doped BiOCl monolayers, calculated using the PBE functional. The dashed line shows the Fermi level that is shifted to zero. There are fewer impurity states around Fermi level for Mn-doped $\mathrm{BiOCl}$, compared to other transition metal doped $\mathrm{BiOCl}$ monolayer. 


\section{Reference}

(1) Peterson, A. A.; Abild-Pedersen, F.; Studt, F.; Rossmeisl J., Nørskov, J. K. How Copper Catalyzes the Electroreduction of Carbon Dioxide into Hydrocarbon Fuels. Energy Environ. Sci. 2010, 3, $1311-1315$.

(2) Zheng, Q.; Chu, W.; Zhao, C.; Zhang, L.; Guo, H.; Wang, Y.; Jiang, X.; Zhao, J. Ab Initio Nonadiabatic Molecular Dynamics Investigations on the Excited Carriers in Condensed Matter Systems. WIREs Comput. Mol. Sci. 2019, 9, e1411.

(3) Chase Jr, M. W. NIST-JANAF thermochemical tables, American Chemical Society, American Institute of Physics for the National Institute of Standards and Technology, Washington, DC, New York, 1998.

(4) Tully, J. C. Molecular Dynamics with Electronic Transitions. J. Chem. Phys. 1990, 93, 1061-1071.

(5) Jaeger, H. M.; Fischer, S.; Prezhdo, O. V. Decoherence-Induced Surface Hopping. J. Chem. Phys. 2012, 137, 22A 545 .

(6) Akimov, A. V.; Prezhdo, O. V. The PYXAID Program for Non-adiabatic Molecular Dynamics in Condensed Matter Systems. J. Chem. Theory Comput. 2013, 9, 4959-4972.

(7) Reuter, K.; Scheffler, M. Composition, Structure, and Stability of $\mathrm{RuO}_{2}$ (110) as a Function of Oxygen Pressure. Phys. Rev. B 2001, 65, 035406. 